\title{
Cytoskeleton localization in the sperm head prior to fertilization
}

\author{
Kateřina Dvořáková, Harry D M Moore ${ }^{1}$, Nataša Šebková and Jiří Paleček \\ Department of Developmental Biology, Faculty of Science, Charles University of Prague, Vinicna 7, 12844 \\ Prague 2, Czech Republic and ${ }^{1}$ Department of Molecular Biology and Biotechnology, University of Sheffield, \\ Sheffield S10 2TN, UK
}

Correspondence should be addressed to K Dvorakova; Email: k_dvorak@natur.cuni.cz

\begin{abstract}
Three major cytoskeletal proteins, actin, tubulin and spectrin, are present in the head of mammalian spermatozoa. Although cytoskeletal proteins are implicated in the regulation of capacitation and the acrosome reaction (AR), their exact role remains poorly understood. The aim of this study was to compare the distribution of the sperm head cytoskeleton before and after the AR in spermatozoa representing a range of acrosome size and shape. Spermatozoa from the human and three rodents (rat, hamster and grey squirrel) were fixed before and after the AR in appropriate medium in vitro. Indirect immunofluorescent localization of cytoskeletal proteins was undertaken with antibodies recognizing actin, spectrin and $\alpha$-tubulin. Preparations were counterstained with propidium iodide and examined by epifluorescent and confocal microscopy. Our results clearly demonstrated changes in localization of cytoskeleton during the AR, mainly in the apical acrosome with further changes to the equatorial segment and post-acrosomal regions. The pattern of cytoskeletal proteins in the sperm head of all the species was similar in respect to various sub-compartments. These observations indicated that the sperm head cortical cytoskeleton exhibits significant changes during the AR and, therefore, support the image of cytoskeletal proteins as highly dynamic structures participating actively in processes prior to fertilization.
\end{abstract}

Reproduction (2005) 130 61-69

\section{Introduction}

The morphology of the head of the mammalian spermatozoon can vary significantly (see Yanagimachi 1981, Eddy \& O'Brien 1994) and species display a wide variety of sperm head shapes and features. Under various selective pressures, spermatozoa evolve in order to benefit their biological functions in the male or female genital tracts and during fertilization. Co-evolution of the male and female gamete leads to further cellular and molecular diversification and reproductive isolation (Bedford 1991, Swanson \& Vacquier 2002). This relatively rapid evolution means that, in most cases, the significance of the morphology of a particular sperm head of a species has remained obscure. Although the shape and relative size of the sperm head may vary considerably, basic organelles such as the apical acrosome, equatorial segment and postacrosomal region are invariably present and these structures can be identified readily in spermatozoa of most mammals. This may indicate that critical constitutive aspects of the function of the sperm head have been conserved (e.g. capacitation, acrosome reaction (AR) and sperm-egg fusion). In contrast, other aspects of sperm function such as motility and sperm transport through the female tract may be subject to greater evolutionary adaptation and might have resulted in the large morphological differences; for example, acrosome size (e.g. squirrel sperm) or some other bizarre feature (e.g. ventral sperm head hooks of plains rat or wood mouse (Breed et al. 2000, Moore et al. 2002)).

The cytoskeleton of the sperm head consists of the resistant structural proteins of the nucleus, and perinuclear theca, which are thought to be responsible, at least in part, for shaping the nucleus (Oko 1998), and the more dynamic proteins of the cortical cytoskeleton. As well as a morphological function, the latter is believed to be involved in cell signalling processes, where data suggest that actin polymerization may represent an important regulatory pathway which is associated with tyrosine phosphorylation in sperm (Brener et al. 2003, Seligman et al. 2004). In general, cytoskeletal proteins include actin, actin-binding proteins such as spectrin, various tubulins (e.g. $\alpha-, \beta$-, $\gamma$-tubulin) and others. In somatic cells, actin filaments lying beneath the plasma membrane form a cortical network involved in functions such as 
cell-to-cell contact and adhering to extracellular matrix, cell growth and migration, and exocytosis. In spermatozoa, a number of cytoskeletal proteins are implicated in key events such as capacitation and the AR (Yanagimachi 1981, Virtanen et al. 1984, Brucker \& Lipford 1995, Brewis \& Moore 1997, Howes et al. 2001, Moore 2001, Brener et al. 2003). For instance, actin may play a role in the AR (Vogl 1989, Palecek et al. 1999) and inhibition of actin polymerization by cytochalasin B and D blocked induction of the human AR by zona pellucida (Liu et al. 1999, Dvorakova et al. 2001). In contrast, phorbol myristate acetate was shown to induce a dramatic change in the ultrastructure of the human acrosome region, termed acrosomal ruffling, which is believed to involve actin polymerization (Liu et al. 2002, Brener et al. 2003). Recently, confocal and electron microscopic studies of boar spermatozoa confirmed that actin, spectrin and tubulin-containing structures are altered after the AR and that cytoskeletal inhibitors influence this event (Peknicova et al. 2001, Dvorakova et al. 2001, Brener et al. 2003).

Four main sperm head types can be readily distinguished in mammals (Yanagimachi 1981). These are a spatulate shape (e.g. boar, bull and rabbit), an ovoid shape (e.g. human and other primates), a falciform shape (e.g. most rodents) and spermatozoa with large acrosomal regions (e.g. guinea pig and grey squirrel). Cross-species comparisons may provide important clues as to the function of cytoskeletal proteins during the fertilization processes and in shaping the sperm head. However, the relationship between sperm head morphology, cytoskeletal elements and events, such as capacitation and the $A R$, have not been investigated in detail with the exception of spatulate-shaped boar spermatozoa (Yagi \& Paranko 1995, Dvorakova et al. 2001, Peknicova et al. 2001). We report here on the localization of cytoskeletal proteins before and after induction of the $A R$ in vitro in representative sperm types, namely spermatozoa of the human, the laboratory rat, the hamster and the grey squirrel.

\section{Materials and Methods}

\section{Animals}

The following male animals were used for our experiments: rat (Wistar), golden hamster (Mesocricetus auratus) and grey squirrel (Sciurus carolinensis). Golden hamsters and rats were maintained and housed at the Field Laboratories, University of Sheffield. Grey squirrel spermatozoa were kindly provided by Mr Mark Ferryman, The Forestry Commission, Wreclesham, Surrey, UK.

\section{Ethics of experimentation}

Ethical permission to use donated human sperm for research was given by the South Sheffield Research Ethics Committee and consent was also obtained from patients.
All animal procedures were carried out in strict accordance with the Animal (Scientific Procedure) Act 1986, and subjected to review by the local ethics committee. Animals were killed by lethal injection $(2 \mu \mathrm{l} / \mathrm{g})$ of sodium pentobarbitone.

\section{Reagents}

All chemicals were purchased from Sigma Chemicals Ltd, UK unless otherwise stated.

\section{Collection and preparation of spermatozoa}

Human sperm ejaculates were obtained from donors of proven fertility attending the Andrology Laboratory, Jessop Hospital, Sheffield, UK. All samples were in the normal range for sperm concentration, motility and morphology as determined by standard protocols (WHO 1992). Spermatozoa of the rat, golden hamster and grey squirrel were collected from the cauda epididymis of adult males.

Spermatozoa were recovered from the cauda epididymis by puncturing epididymal tubules and releasing spermatozoa in phosphate-buffered saline (PBS) for 10 min. The suspension of both epididymal and human ejaculated sperm was washed twice in PBS. Sperm motility was examined by an inverted microscope with a thermostatically controlled stage at $37^{\circ} \mathrm{C}$. The $A R$ was induced after the incubation of spermatozoa by the addition of the calcium ionophore A-23187 at a final concentration of $2 \mu \mathrm{M}$, followed by a 30-min incubation at $37^{\circ} \mathrm{C}$ in $5 \% \mathrm{CO}_{2}$ in air (Moos et al. 1993). In order to evaluate whether sperm had undergone the AR, lectin PNA conjugate Alexa Fluor 488 (L-21409; Molecular Probes, Prague, Czech Republic) was used. Research has shown PNA to be selective for acrosomes in the sperm of rat, human, hamster and others (Calvo et al. 1995, Fierro et al. 1996, Aviles et al. 1997). PNA solution of a final concentration of $1 \mu \mathrm{l} / \mathrm{ml}$ was added to the sperm suspension and the status of the acrosome in 200 sperm was examined under the epifluorescent microscopy. Drops of spermatozoa from the stock were aspirated gently by glass pipette onto a glass slide, smeared, air-dried and fixed for immunocytochemistry. For spectrin and $\alpha$-tubulin immunolocalization, spermatozoa were fixed with $100 \%$ methanol for $8 \mathrm{~min}$ and $100 \%$ acetone for $6 \mathrm{~min}$ at $-20^{\circ} \mathrm{C}$. For actin immunolocalization, spermatozoa were fixed with $4 \%$ formaldehyde in PBS at $22^{\circ} \mathrm{C}(45 \mathrm{~min})$ then washed in PBS, rinsed with distilled $\mathrm{H}_{2} \mathrm{O}$ and air-dried. Adequate control slides were run simultaneously through the whole experiment.

\section{Indirect immunofluorescent localization}

Slides with fixed spermatozoa were rinsed with PBS and, after 1 -h blocking with $3 \%$ bovine serum albumin (BSA) in PBS, incubated for $1 \mathrm{~h}$ at $37^{\circ} \mathrm{C}$ with primary antibodies against G- and F-actin (A2668), spectrin (S1515), $\alpha$-tubulin (T9026), or without BSA blocking, and at room 
temperature with fungal toxin Phalloidin-Fluorescein Isocyannate (FITC; P5282). Antibodies were diluted in 3\% BSA. After washing in PBS, slides were incubated for a further hour with secondary antibody FITC conjugate sheep anti-mouse IgG (F5387) or goat anti-rabbit IgG (F7512). Alexa Fluor 594 donkey anti-rabbit IgG (A21207; Molecular Probes) was used to detect spectrin in spectrinactin co-localization experiments. After repeated washing in PBS, slides were mounted in Vectashield mounting medium with or without propidium iodide (Vector Laboratories, Prague, Czech Republic) for counterstaining the nucleus. Slides were routinely examined by epifluorescent microscope. The photomicrographs shown were taken by confocal microscope Leica TCS 4D, with a tunable 457$675 \mathrm{~nm}$ laser excitation and digital image capture (Fig. 2) or Leica TCS SP2 RS high-speed confocal/two photon system for live cell imaging and dynamics (Figs. 3 and 4).

\section{Immunoblot analysis}

The biochemical specificity of primary antibodies was examined by SDS-electrophoresis and immunoblotting technique. SDS-PAGE was performed according to the method of Laemmli (1970), Western blot was done according to Towbin et al. (1979). Briefly, sperm samples were washed twice with $\mathrm{PBS}$, resuspended in non-reducing lysis buffer and heated to $100^{\circ} \mathrm{C}$ for $5 \mathrm{~min}$. A protein equivalent of $10^{6}$ cells was loaded into wells of an SDSPAGE gel. Precision Plus Protein All Blue Standards (BioRad, Nad ostrovem, Prague, Czech Republic) were used as molecular weight markers. Separated proteins were transferred electrophoretically onto nitrocellulose membrane and detected by incubation with a primary antibody, followed by a secondary antibody, either antimouse IgG (A0168) or anti-rabbit (A0545) IgG peroxidase conjugate. Non-specific sites were blocked with PBS blocking solution (5\% skim milk and 0.05\% Tween 20). Protein bands were visualized by enhanced chemiluminescence (Amersham Biosciences, Amersham, Bucks, UK).

\section{Results}

For clarity, the comparative localization of cytoskeleton changes in the sperm head in control and after the AR induction is described for each protein investigated. The fluorescent staining of comparative localization is shown in Table 1. For each analysed group, after optimizing working conditions, 200 cells were examined in three parallel experiments. Results given represent approximately $90 \%$ of the population. The status of the acrosome after the calcium ionophore-induced AR was examined with PNA lectin in 200 cells for each group and experiment. If a minimum of $95 \%$ of sperm had undergone the $A R$, then the population of sperm was accepted for further experiments. Parallel controls with untreated sperm were run for each experiment.
Table 1 Localization of cytoskeletal proteins in sperm head control and after the AR. In the case of actin, data show summarized results, obtained by both antibody anti-G- and F-actin and phalloidin-FITC conjugate.

\begin{tabular}{llccc}
\hline Protein & Species & Acrosome & ES & PA \\
\hline \multirow{2}{*}{ Actin } & Human & $+/ \pm$ & $\pm /+$ & $+/+$ \\
& Rat & $+/ \pm$ & $\pm /+$ & $+/+$ \\
& Hamster & $+/ \pm$ & $\pm /+$ & $+/+$ \\
\multirow{3}{*}{ Spectrin } & Squirrel & $+/ \pm$ & $+/+$ & $+/+$ \\
& Human & $-/-$ & $+/+$ & $+/+$ \\
& Rat & $+/-$ & $+/+$ & $+/+$ \\
& Hamster & $+/-$ & $+/+$ & $+/+$ \\
$\alpha$-tubulin & Squirrel & $+/-$ & $+/+$ & $+/+$ \\
& Human & $-/ \pm$ & $+/+$ & $+/+$ \\
& Rat & $-/-$ & $\pm /+$ & $+/+$ \\
& Hamster & $+/-$ & $\pm /+$ & $+/+$ \\
& Squirrel & $+/-$ & $+/+$ & $+/+$
\end{tabular}

Fluorescence signal: positive $(+)$; absent $(-)$ and weak $( \pm)$. ES, equatorial segment; PA, post-acrosomal region.

\section{Specificity of antibodies}

Immunofluorescence was not detected on controls without primary antibody, which were run throughout the whole experiment (not shown). The specificity of antibodies was also proved by pre-incubation of antibodies with authentic antigens, anti-actin, spectrin and $\alpha$-tubulin, which blocked the immunostaining. Immunoblotting of proteins extracted from human and rodent sperm samples showed clear bands at the relative molecular weights for the corresponding antigen (actin, $42 \mathrm{kDa} ; \alpha$ - and $\beta$-spectrin, 240 and $220 \mathrm{kDa}$; $\alpha$-tubulin, $55 \mathrm{kDa}$ ) (Fig. 1, rat sperm extract). Equivalent bands were detected for all species indicating similar cross-species specificity. The specificity of the polyclonal antibody for G- and F-actin was stressed by results obtained with Phalloidin-FITC or Alexa Fluor conjugate, which binds specifically to F-actin only.

\section{Localization of cytoskeletal proteins in the sperm head}

Representative micrographs of studied cytoskeletal proteins in control spermatozoa and after the calcium ionophore-induced AR are presented in Figs. 2 and 3, and summarized in Table 1.

\section{Actin}

There were similarities in the localization of actin in the sperm head for each selected species before treatment (Figs. 2a and 3a and 3c). Bright immunofluorescent staining was seen in the entire region of the apical acrosome, covering the cortical cytoskeleton between the plasma membrane and the outer acrosomal membrane, and the sub-acrosomal cytoskeleton between the inner acrosome membrane and the nuclear envelope. In spermatozoa of the grey squirrel, the cortical cytoskeleton of apical acrosome displayed a spotty localization and, besides the staining in equatorial segment, there was another actin 


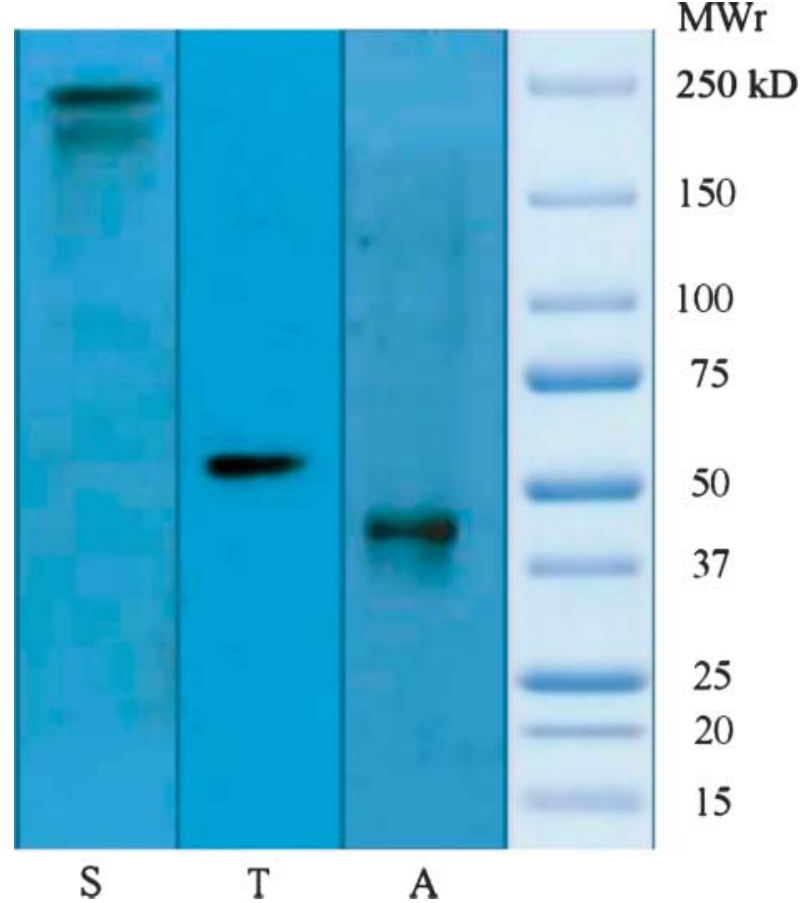

Figure 1 Immunoblotting analysis of rat spermatozoa extract for $(\mathrm{S})$ spectrin ( $\alpha$ and $\beta$ ), 240 and $220 \mathrm{kDa}$, (T) $\alpha$-tubulin, $55 \mathrm{kDa}$ and (A) actin, $42 \mathrm{kDa}$. Analysis for the remaining rodent and human sperm was the same as for the rat (not shown). MWr; Molecular weight marker.

ring seen in the post-acrosomal region. Several changes were observed after the AR. Generally, the most prominent became the equatorial segment that was clearly stained in all species studied (Figs. $2 \mathrm{~b}$ and $3 \mathrm{~b}$ and $3 \mathrm{~d}$ ). In squirrel sperm after the AR, the inner acrosome membrane had a punctuate appearance compared with untreated sperm (Fig. 2b). The pattern given by staining with Phalloidin-FITC (Fig. 3) was quite different in control spermatozoa. The anti-actin antibody detected intense staining in the entire region of the apical acrosome, even between the plasma membrane and the acrosomal membrane. In contrast, Phalloidin detected F-actin enriched in the acrosomal region, clearly defining the shape of the acrosome vesicle (Fig. 3c and 3e). Generally in human sperm, the distribution of actin cytoskeleton in the postacrosomal region was weaker in control sperm than in those after AR which is best seen in the pictures without propidium iodide nuclear staining (Fig. 3). In rodent spermatozoa after the AR, actin localization became prominent in the very distal apical region, which contains paraacrosomal cytoskeleton (Figs. $2 \mathrm{~b}$ and $3 \mathrm{~d}$ and 3f). However, the labelling was present in control sperm as well (Fig. 2a and 3c and 3e).

In order to obtain the sharpest picture of the sperm head, which was our main interest, we sometimes merged only selected sections obtained by confocal microscopy. These sections did not necessarily have to cover the plane of the tail; therefore the staining of the tail; could sometimes be seen as weak or not present.

\section{Spectrin}

The staining pattern for spectrin was similar to the one for actin in certain aspects. This protein was present in the acrosome domain of rodent sperm head (Figs. 2c and 4b). In human spermatozoa, there was discrete bright immunofluorescence of the apical equatorial segment without a strong labelling of cortical cytoskeleton lying between the plasma and outer acrosomal membrane. Localization in the equatorial segment similar to the one seen in actin was apparent again in squirrel spermatozoa (Fig. 2c). After the $A R$, the pattern in human spermatozoa in the equatorial segment remained mainly unchanged; however, a slight increase in labelling over the post-acrosomal region was seen (Fig. 2d). Localization of spectrin in rodent spermatozoa was similar to the one for actin in the equatorial segment, with a stronger immunostaining in the post-acrosomal region (Fig. 2d). Para-acrosomal cytoskeleton in rat and hamster sperm head was strongly labelled both before (Figs. 2c and 4b) and after the AR (Fig. 2d).

The double immunofluorescence staining technique confirmed that spectrin and F-actin co-localize with each other (Fig. 4, control rat spermatozoa only shown). In human spermatozoa before the $A R$, spectrin and F-actin co-localized only in the equatorial segment and the apical peri-nuclear theca, due to the fact that in control human spermatozoa spectrin was not detected in the acrosome. In addition we ran fluorescent resonance energy transfer analysis, which confirmed the co-localization of these two proteins in the above-mentioned organelles of control and acrosome-reacted sperm (K Dvorakova \& F Difato, unpublished results).

\section{$\alpha$-Tubulin}

Prior to the AR, the apical acrosome cytoskeleton and equatorial segment in human and all rodent species displayed some fluorescence (Fig. 2e). In human sperm, a ring in the middle of the apical acrosome was prominent. Fluorescence was also seen in the post-acrosomal region, especially in hamster sperm where strong well-defined dorsal and ventral localization was observed. After the AR (Fig. 2f), the equatorial segment became more crisply defined but in the sub-acrosomal layer the faint fluorescence was seen only in the grey squirrel sperm. The para-acrosomal and post-acrosomal regions remained unchanged essentially in all falciform head sperm types. (Fig. 2e and 2f).

\section{Discussion}

The objective of this study was to examine the microfilamental and microtubular cytoskeleton of the sperm head in relation to the ionophore-induced $A R$. The relocation of cytoskeletal proteins following the $A R$ is likely to be important for subsequent events of fertilization, such as sperm-zona binding, sperm penetration of the zona 

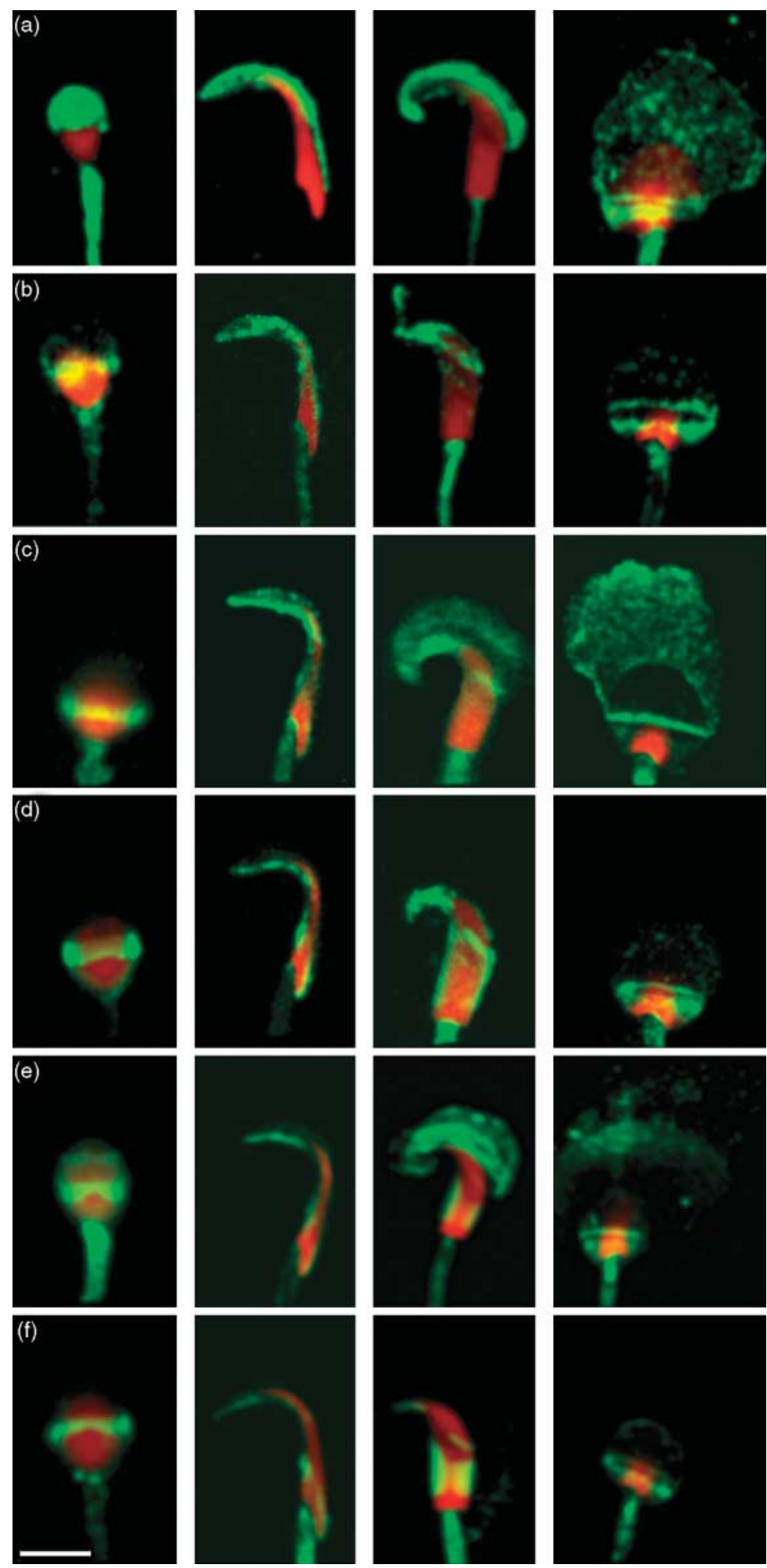
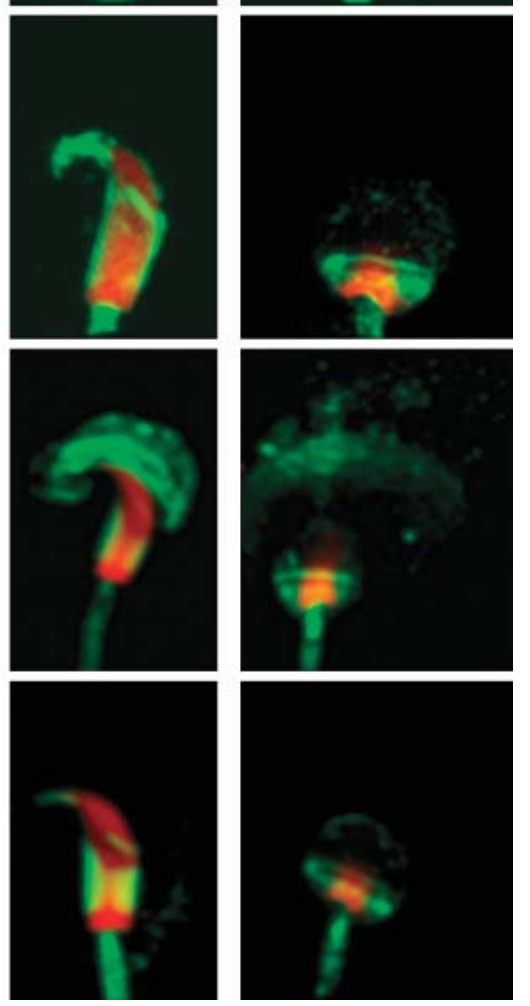

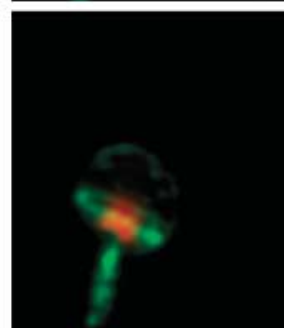

Figure 2 Immunofluorescent localization of cytoskeletal proteins in the sperm head of human, rat, hamster and grey squirrel spermatozoa (left to right). Actin (a) control and (b) after AR, spectrin (c) control and (d) after AR and $\alpha$-tubulin (e) control and ( $\mathrm{f}$ ) after AR. Red is propidium iodide staining of the nucleus; green corresponds to the cytoskeletal proteins. Scale bar $=5 \mu \mathrm{m}$. pellucida or sperm-egg fusion (Brewis \& Moore 1997, Primakoff \& Myles 2002). Here, we were interested in comparing generic aspects of cytoskeletal localization in mammalian spermatozoa with the more specific features related to morphology, since it is clear that the kinetics of the AR in terms of vesiculation of membrane and loss of contents varies considerably between species (Yanagimachi 1981). It was surmised that by carefully comparing spermatozoa with different head shape one might ascertain whether antigen localization patterns reflected primarily 


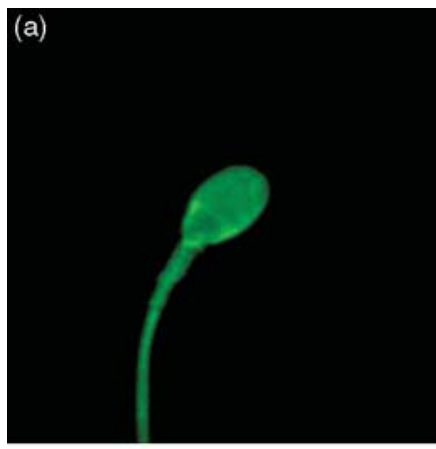

(b)
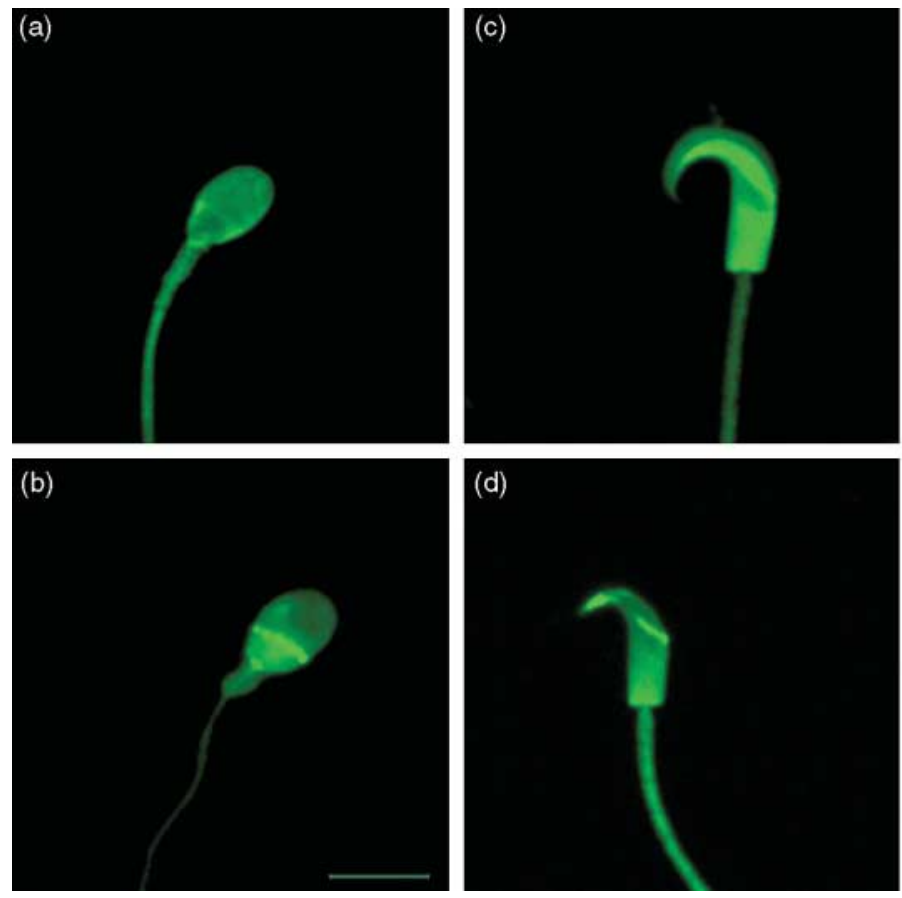
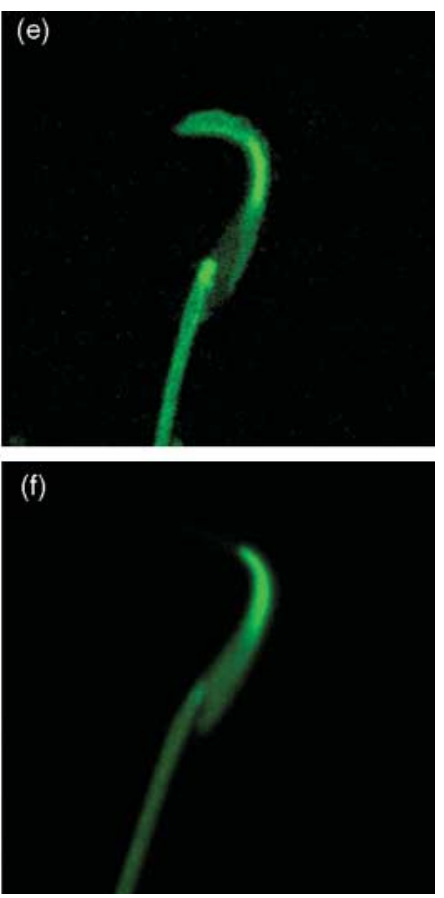

Figure 3 Immunofluorescent localization of F-actin by Phalloidin-FITC in the sperm head of human (a) control and (b) after AR, hamster (C) control and (d) after AR and rat (e) control and (f) after AR. Scale bar $=5 \mu \mathrm{m}$. morphological or functional features of the cell. The cytoskeletal proteins selected were the same as those used in a previous study with boar spermatozoa (representing the spatulate morphology), where it was shown that these proteins were redistributed during and after the AR (Dvorakova et al. 2001). The AR was induced with calcium ionophore to produce a consistent population of cells which had undergone the competed AR (Jaiswal et al. 1999).
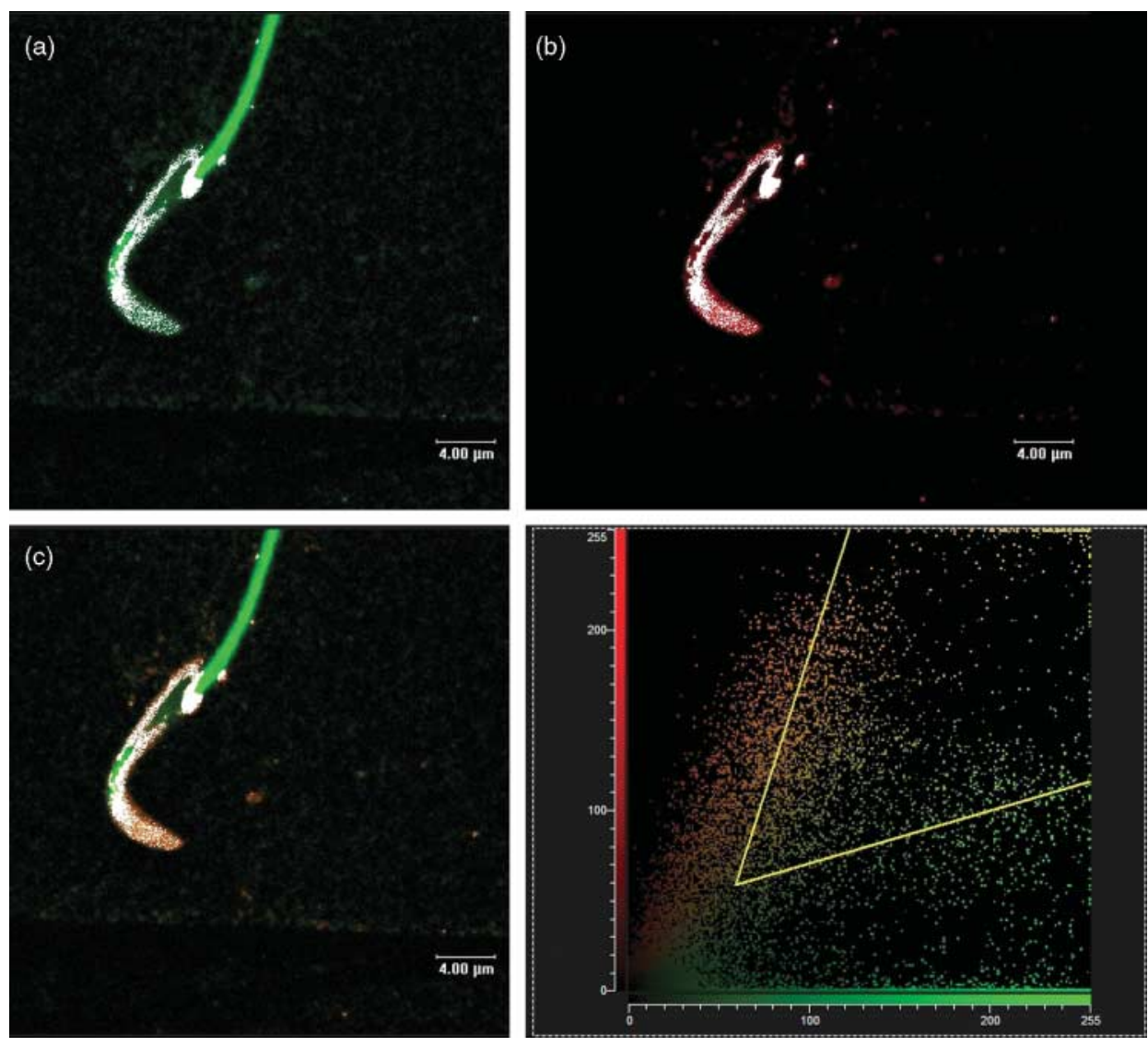

Figure 4 Co-localization of F-actin and spectrin in control spermatozoa; the white marking represents the co-localization of both proteins, which is defined by the graph; (a) F-actin, (b) spectrin and (c) merged. Green corresponds to F-actin; red corresponds to spectrin. In the graph, the co-localization of two proteins is defined by the trapezoid lying between the $x$ and y axes. Scale bars $=4 \mu \mathrm{m}$. 
The presence of actin within the head of the selected spermatozoa has been confirmed previously in several studies, although the exact distribution has been shown to vary, possibly because of the methods of fixation, antibody specificity, sensitivity of technique and membrane permeabilization (Fouquet et al. 1990, Fouquet \& Kann 1992). The reasons for choosing anti-actin and spectrin polyclonal antibodies for this study was their convenient cross-reactivity and therefore consistency in immunohistochemical testing across all selected species, and also because of obtaining consistent results with previous testing of boar spermatozoa representing spatulate sperm head shape (Dvorakova et al. 2001). We also used fungal toxin Phalloidin conjugated with FITC for the exclusive staining of polymerized F-actin microfilaments. The results obtained by Phalloidin-FITC in control sperm were quite different from those obtained by polyclonal anti-actin antibody. In control sperm, anti-actin antibody detected the entire apical acrosome, even between the plasma and the outer acrosomal membrane; however, in contrast, F-actin, detected by Phalloidin, was clearly confined to the acrosome region. This difference could be explained by the pool of G-actin localized between the plasma membrane and the acrosomal membrane, which would only be detected by the anti-actin antibody. These results contrast somewhat with the investigation of Howes et al. (2001), where actin and actin-binding proteins were localized to just the margin of the anterior acrosomal and post-acrosomal region of mature epididymal bovine spermatozoa and relocated to a postacrosomal region after the AR. However, the relocalization of F-actin to the post-acrosomal region in boar ejaculated sperm after the AR described by Howes et al. (2001) correlates with our results on human sperm after the AR; however, the weak labelling in this region was seen in control sperm as well. This clear redistribution of F-actin in the post-acrosomal region was not seen in rodent sperm, and may be due to the fact that samples of rodent sperm were obtained from the epididymis. Nevertheless, in agreement with the studies of Brener et al. (2003) and Paranko et al. (1994), the post-acrosomal region in control sperm showed the presence of $\mathrm{F}$-actin, and this was in contrast to Howes et al. (2001). The polymeric form of actin (F-actin) is thought to be particularly important as its depolymerization between the outer acrosomal and plasma membrane is correlated with initiation of the AR in studies on guinea pig (Breitbart \& Spungin 1997) and on bull, ram, mouse and human sperm (Brener et al. 2003). In this respect, the spermatozoa of the grey squirrel represents the closest comparison as they have a massive acrosome, and actin was clearly localized mainly to the cortical cytoskeleton between the plasma and outer acrosomal membrane in control spermatozoa. A punctuate appearance over the acrosomal region suggested that there were focal points of actin along the membrane. The wider filamentous network might have been observed in spermatozoa which were capacitated (Brener et al. 2003). This would be in correlation with our findings on the localization of a monomeric form of actin between the plasma membrane and the outer acrosomal membrane in control spermatozoa. This pool of G-actin could therefore be polymerized during the capacitation; however, this was not the aim in this study. These observations suggested that actin does not serve only as a structural cytoskeletal protein to maintain shape but may also be involved in the events prior to fertilization, such as the AR.

After induction of the $A R$, the relative distribution of actin seemed to be related to the type of reaction. In both the squirrel and the hamster, the AR is characterized by rapid divestment of the entire acrosomal cap. In these species, actin remained localized under the inner acrosomal membrane and was more prevalent at the apical margin of the equatorial segment. Moreno-Fierros et al. (1992) showed a similar redistribution of F-actin in guinea pig spermatozoa. For human and rat spermatozoa there was noticeably more actin localized on vesiculated acrosomal membrane. This may correspond to actin playing a role in maintaining the presence of vesiculated membranes after the AR to aid in the tethering of the fertilizing spermatozoa to the zona pellucida. Generally, the most prominent became the equatorial segment after the AR induction in all species studied, and the apical acrosome showed a decrease of actin labelling, which is in agreement with the findings of both Howes et al. (2001) and Brener et al. (2003). Moreover Yagi \& Paranko (1995) suggested that lateral prongs may be a merger zone for actin and two other actin-binding proteins such as $\alpha$-actinin and spectrin.

The role of actin-binding proteins has also been investigated previously by Virtanen et al. (1984) who suggested that spectrin and vimentin can be in correlation with distinct surface specializations of the sperm cells. These findings are in agreement with our results which demonstrated the co-localization of spectrin with actin in both control and acrosome-reacted sperm, with the exception of human spermatozoa before the $A R$, where spectrin co-localized only in the equatorial segment and the apical peri-nuclear theca. In addition, perforatorium, containing a para-acrosomal cytoskeleton, and post-acrosomal regions stained for spectrin, in the case of rat and hamster sperm. Its presence in the acrosomal plasma membrane has also been confirmed by results from electron microscopy observations (Hernandez-Gonzalez et al. 2000). Interestingly, even though squirrel sperm exhibited actin at the proximal and distal margin of the equatorial segment in both control sperm and acrosome-reacted sperm, in the case of spectrin only one narrow band across the equatorial segment was seen in control sperm. However, after AR induction the second band in the postacrosomal region was revealed. This may suggest the relocalization of spectrin during the AR due to its structural role in the stabilization of the equatorial segment as suggested by Yagi \& Paranko (1995). Besides spectrin possibly having a structural role, there is a possibility that it also plays an active role during sperm-egg fusion (Yagi 
\& Paranko 1995) or even during capacitation and the AR by its ability to form the spectrin/calmodulin complex that involves $\mathrm{Ca}^{2+}$ as a mediator (Virtanen et al. 1984).

There is plenty of evidence for the presence of tubulins in spermatocytes and spermatids during mammalian spermatogenesis (Moreno \& Schatten 2000, Kierszenbaum 2002, Kato et al. 2004) and also in mature spermatozoa (Draber et al. 1991, Dvorakova et al. 2001, Peknicova et al. 2001). However, because of the large diversity in the tubulin superfamily there is a gap in the field covering spermatogenesis and mature sperm undergoing changes prior to fertilization. Most eukaryotic cells can express multiple isotypes of $\alpha$ - and $\beta$-tubulin and this diversity is further expanded by a number of post-translational modifications (Luduena 1998). Although the functional significance of tubulin diversity is still difficult to associate with consistent biological functions, there is increasing evidence that different $\alpha$ - and $\beta$-tubulin isotypes and post-translational modification can impact microtubule structure and function (Kierszenbaum 2002).

We have here reported the obvious differences in the localization of staining of the $\alpha$-tubulin suggesting that these proteins may be related to morphology as well as function. In squirrel, and hamster control sperm, $\alpha$-tubulin was clearly localized within the cortical cytoskeleton of the apical acrosome region. These cortical microtubules might be a network which was originally distributed in the cortex of spermatocytes and later helped with forming the acrosomal vesicle by being attached to Golgi apparatus (Moreno \& Schatten 2000). In human spermatozoa, $\alpha$-tubulin was displayed as a distinct band within the apical acrosome, suggesting a restricted distribution of this protein in the apical peri-nuclear theca between the inner acrosomal and nuclear membranes. This might be in correlation with the previously reported localization of $\alpha$-tubulin by Moreno \& Schatten (2000), forming a bundle running around the nucleus in round spermatids, as it seems that tubulins persist in the sperm head of mature spermatozoa. However, in rat spermatozoa, the para-acrosomal cytoskeleton was mainly stained. Certainly, in the squirrel, the localization of $\alpha$-tubulin represented adjacent regions of the apical acrosome. Interestingly, this region in boar spermatozoa exhibited distinct changes in the presence of inhibitors such as vinblastine (Dvorakova et al. 2001). After the AR, $\alpha$-tubulin was restricted mainly to the apical equatorial margin and post-acrosomal region, which was especially interesting in the case of hamster sperm, where its ventral and dorsal part became distinct compared with the control. Similar findings on human and boar sperm on the redistribution of $\beta$-tubulin in the sperm head due to the AR were published by Draber et al. (1991) and Peknicova et al. (2001); however, Virtanen et al. (1984) reported no staining with anti-tubulin antibodies in the head of human sperm, only in the neck region and entire tail. Nevertheless, Whyard et al. (2000) found that the regulatory subunit (RS) of the RNA binding protein (RBP) which is $20 \mathrm{kDa}$ microtubule-associated protein, was present besides the tail localization in human and rat sperm and also in the rat sperm head. Moreover, its distribution co-localized with $\beta$-tubulin. The comparison between our study on $\alpha$-tubulin and others on $\beta$-tubulin (Draber et al. 1991, Peknicova et al. 2001) is appropriate as the tubulin is a heterodimer consisting of $\alpha$ - and $\beta$-tubulin subunits, which is nearly ubiquitous among eukaryotic cells. It is relevant to assume that the presence of $\alpha$-tubulin is accompanied by the presence of a $\beta$-tubulin subunit at the same time. There are different forms of tubulin but they are very similar, and will generally polymerize into mixed microtubules in vitro (Chakraborty et al. 1999).

In summary, the selected cytoskeletal proteins were mostly localized to the apical and the equatorial acrosomal region of the sperm head. While the intensity of staining differed between the sperm types, there was a general similarity in the localization patterns in control sperm and after the AR induction. Hence, these results suggested that cytoskeletal proteins in mature spermatozoa, at least, may play more of a role in the development of the AR and priming the spermatozoa for other fertilization events than in maintaining distinctive head morphologies. The modified localization of cytoskeletal proteins within the equatorial segment of the acrosome after the AR may be significant. This region is altered in response to the AR and the overlying plasma membrane becomes capable of fusing with the egg membrane at gamete fusion. It is likely that cytoskeletal proteins would be important at this time to reorganize membrane domains into fusogenic regions.

\section{Acknowledgements}

We are grateful to Nick Jenkins for technical assistance. The work was funded by the GACR no. 204/02/1373 and MSMT no. 0021620828 . The authors declare that there is no conflict of interest that would prejudice the impartiality of this scientific work.

\section{References}

Aviles M, Castells MT, Martinez-Menarguez JA, Abascal I \& Ballesta J 1997 Localization of penultimate carbohydrate residues in zona pellucida and acrosomes by means of lectin cytochemistry and enzymatic treatments. Histochemical Journal 29 583-592.

Bedford JM 1991 coevolution of gametes. In A Comparative Overview of Mammalian Fertilization, pp 3-50. Eds BS Dunbar \& MG O'Rand. New York: Plenum Press.

Breed WG, Idriss D \& Oko RJ 2000 Protein composition of the ventral processes on the sperm head of Australian hydromyine rodents. Biology of Reproduction 63 629-634.

Breitbart H \& Spungin G 1997 The biochemistry of the acrosome reaction. Molecular Human Reproduction 3 195-202.

Brener E, Rubinstein S, Cohen G, Shternall K, Rivlin J \& Breitbart H 2003 Remodeling of the actin cytoskeleton during mammalian sperm capacitation and acrosome reaction. Biology of Reproduction 68 837-845.

Brewis I \& Moore HDM 1997 Molecular mechanisms of gamete recognition and fusion at fertilization. Human Reproduction $\mathbf{1 2}$ (Suppl. 11) 156-165. 
Brucker C \& Lipford GB 1995 The human sperm acrosome reaction: physiology and regulatory mechanisms. An update. Human Reproduction Update $151-62$.

Calvo A, Pastor LM, Horn R \& Pallares J 1995 Histochemical study of glycoconjugates in the epididymis of the hamster (Mesocricetus auratus). Histochemical Journal 27 670-680.

Chakraborty S, Sarkar N \& Bhattacharyya B 1999 Nucletide-dependent bisANS binding to tubulin. Biochimica et Biophysica Acta 1432 350-355.

Draber P, Draberova E \& Viklicky V 1991 Immunostaining of human spermatozoa with tubulin domain-specific monoclonal antibodies. Recognition of a unique beta-tubulin epitope in the sperm head. Histochemistry 95 519-524.

Dvorakova K, Palecek J \& Peknicova J 2001 Changes in immunocytochemical localization of cytoskeletal proteins in boar spermatozoa after the AR induced by specific cytoskeletal inhibitors. Folia Biologica 47 18-27.

Eddy EM \& O'Brien DA 1994 The spermatozoon. In The Physiology of Reproduction, edn 5, pp 29-77. Eds E Knobil \& JD Neill. New York: Raven Press.

Fierro R, Foliguet B, Grignon G, Daniel M, Bene MC, Faure GC \& Barbarino-Monnier P 1996 Lectin-binding sites on human sperm during acrosome reaction: modification judged by electron microscopy/flow cytometry. Archives of Andrology 36 187-196.

Fouquet JP \& Kann ML 1992 Species-specific localization of actin in mammalian spermatozoa. Fact or artifact? Microscopic Research Techniques $20251-258$.

Fouquet JP, Kann ML \& Dadoune JP 1990 Immunoelectron microscopic distribution of actin in hamster spermatids and epididymal, capacitated and acrosome-reacted spermatozoa. Tissue and Cell 22 291-300.

Hernadez-Gonzalez EO, Lecona-Valera AN, Escobar-Herrera J \& Mujica A 2000 Involvement of an F-actin skeleton on the acrosome reaction in guinea pig spermatozoa. Cell Motility and the Cytoskeleton $\mathbf{4 6} 43-58$.

Howes ES, Hurst SM \& Jones R 2001 Actin and actin binding proteins in bovine spermatozoa: potential role in membrane remodeling and intracellular signaling during epididymal maturation and the acrosome reaction. Journal of Andrology 22 62-72.

Jaiswal BS, Eisenbach M \& Tur-Kaspa I 1999 Detection of partial and complete acrosome reaction in human spermatozoa: which inducer and probes to use? Molecular and Human Reproduction 5 214-219.

Kato A, Nagata Y \& Todokoro K $2004 \delta$-Tubulin is a component of intercellular bridges and both the early and mature perinuclear rings during spermatogenesis. Developmental Biology 269 196-205.

Kierszenbaum AL 2002 Sperm axoneme: a tale of tubulin posttranslation diversity. Molecular Reproduction and Development 62 1-3.

Laemmli UK 1970 Cleavage of structural proteins during the assembly of the head of the bacteriophage T4. Nature 227 680-685.

Liu DY, Martic M, Clarke GN, Dunlop ME \& Baker HWG 1999 An important role of actin polymerization in the human zona pellucida-induced AR. Molecular Human Reproduction 5 941-949.

Liu DY, Martic M, Grkovic I, Garrett C, Dunlop ME \& Baker HW 2002 Phorbol myristate acetate induces ruffling of the acrosome of human sperm. Fertility and Sterility $\mathbf{7 8} 128-136$.

Luduena RF 1998 Multiple forms of tubulin: different gene products and covalent modifications. International Review of Cytology 178 207-275.

Moore HDM 2001 Molecular biology of fertilization. Journal of Reproduction and Fertility 57 (Suppl) 105-110.

Moore HDM, Dvorakova K, Jenkins N \& Breed W 2002 Exceptional sperm cooperation in wood mice. Nature 418 174-177.
Moos J, Peknicova J \& Tesarik J 1993 Relationship between molecular conversion of acrosin and the progression of exocytosis in the ionophore-induced AR. Biochimica et Biophysica Acta $\mathbf{1 1 7 6}$ 199-207.

Moreno RD \& Schatten G 2000 Microtubule configurations and posttranslational alpha-tubulin modification during mammalian spermatogenesis. Cell Motility and the Cytoskeleton 46 235-246.

Moreno-Fierros L, Hernandez EO, Salgado ZO \& Mujica A 1992 F-actin in guinea-pig spermatozoa: its role in calmodulin translocation during AR. Molecular Reproduction and Development 33 $172-181$.

Oko R 1998 Occurrence and formation of cytoskeletal proteins in mammalian spermatozoa. Andrologia 30 193-206.

Palecek J, Peknicova J \& Vitu M 1999 Changes in immunochemical localization of cytoskeletal proteins in human and boar spermatozoa before and after AR. Folia Biologica 45 13-20.

Paranko J, Yagi A \& Kuusisto M 1994 Immunocytochemical detection of actin and $53 \mathrm{kDa}$ polypeptide in the epididymal spermatozoa of rat and mouse. Anatomical Record 240 516-527.

Peknicova J, Kubatova A, Sulimenko V, Draberova E, Viklicky V, Hozak P \& Draber P 2001 Differential subcellular distribution of tubulin epitopes in boar spermatozoa: recognition of class III $\beta$-tubulin epitope in sperm tail. Biology of Reproduction 65 672-679.

Primakoff P \& Myles DG 2002 Penetration, adhesion and fusion in mammalian sperm-egg interaction. Science 296 2183-2185.

Seligman J, Zipser Y \& Kosower NS 2004 Tyrosine phosphorylation, thiol status and protein tyrosine phosphatase in rat epididymal spermatozoa. Biology of Reproduction 71 1009-1015.

Swanson WJ \& Vacquier VD 2002 The rapid evolution of reproductive proteins. Nature Genetics 3 137-144.

Towbin H, Staehelin T \& Gordon G 1979 Electrophoretic transfer of proteins from polyacrylamide gels to nitrocellulose sheets. Procedure and some applications. PNAS 76 4354-5350.

Virtanen I, Badley RA, Paasivuo R \& Lehto VP 1984 Distinct cytoskeletal domains revealed in sperm cells. Journal of Cell Biology 99 1083-1091.

Vogl AW 1989 Distribution and function of organized concentration of actin filaments of mammalian spermatogenic cells and Sertoli cells. International Review of Cytology 119 1-56.

WHO 1992 In Laboratory Manual for the Examination of Human Semen and Sperm-Cervical Mucus Interactions, edn 3. Cambridge: Cambridge University Press.

Whyard TC, Cheung W, Sheynkin Y, Waltzer WC \& Hod Y 2000 Identification of RS as a flagellar and head sperm protein. Molecular Reproduction and Development 55 189-196.

Yagi A \& Paranko J 1995 Actin, $\alpha$-actinin, and spectrin with specific associations with the postacrosomal and acrosomal domain of bovine spermatozoa. Anatomical Record 241 77-87.

Yanagimachi R 1981 Mechanism of fertilization in mammals. In Fertilization and Embryonic Development In Vitro, pp 81-182. Eds L Mastroianni \& JD Biggers. New York: Plenum Press.

Received 28 October 2004

First decision 17 December 2004

Revised manuscript received 31 March 2005

Accepted 22 April 2005 